University of Nebraska - Lincoln

DigitalCommons@University of Nebraska - Lincoln

7-1-1995

\title{
A PHOTOMETRIC STUDY OF V508 CYGNI
}

S.N. Goderya

University of Nebraska - Lincoln

Kam-Ching Leung

University of Nebraska-Lincoln, kleung2@unl.edu

Edward G. Schmidt

University of Nebraska-Lincoln, eschmidt1@unl.edu

Follow this and additional works at: https://digitalcommons.unl.edu/physicsschmidt

Part of the Physics Commons

Goderya, S.N.; Leung, Kam-Ching; and Schmidt, Edward G., "A PHOTOMETRIC STUDY OF V508 CYGNI" (1995). Edward Schmidt Publications. 19.

https://digitalcommons.unl.edu/physicsschmidt/19

This Article is brought to you for free and open access by the Research Papers in Physics and Astronomy at DigitalCommons@University of Nebraska - Lincoln. It has been accepted for inclusion in Edward Schmidt Publications by an authorized administrator of DigitalCommons@University of Nebraska - Lincoln. 


\title{
A PHOTOMETRIC STUDY OF V508 CYGNI
}

\author{
S. N. Goderya, K. C. LeUNG, AND E. G. SCHMidT \\ Behlen Observatory, University of Nebraska, Lincoln, Nebraska 68588-0111 \\ Received 1994 December 13; revised 1995 March 24
}

\begin{abstract}
The Behlen observatory $0.76 \mathrm{~m}$ telescope $\mathrm{CCD}$ photometer has been used to obtain nearly 700 observations of the short period eclipsing binary V508 Cyg. These observations were done using $V$ and $R$ bandpass filters on 9 nights in 1992. Previously published light elements and the present eight determinations of eclipse timings are used to determine a new epoch and a more accurate orbital period of 0.7796587 days. The photometric observations and solutions which have been obtained with the 1993 version of the WilsonDevinney model show that V508 Cyg is a W UMa type contact binary system. Analyses give two possible contact solutions of different mass ratios $\left(q=m_{2} / m_{1}\right.$, where star 1 is eclipsed at the primary minimum). One with $q=0.44$ gives an A-type W UMa system configuration, while the other with $q=1.19$ gives a W-type W UMa system configuration. V508 Cyg does not have a spectral classification, however, based on the color ( $V-R=0.566 \mathrm{Schmidt} 1991 \mathrm{~b}$ ), we estimate it to be G5. Generally W UMa systems with spectral-type G5 have periods ranging from 0.25 to 0.5 days. The considerably longer period of V508 Cyg suggests that it may be an evolved contact system with case B mass transfer. Both solutions indicate that the two components have similar temperature and luminosities. It is suspected that this system may be a double lined spectroscopic binary. Therefore it is recommended that spectroscopic observations of V508 Cyg be obtained so that a unique mass ratio can be established. (c) 1995 American Astronomical Society.
\end{abstract}

\section{INTRODUCTION}

The Behlen Observatory variable star survey contains various light curves and associated parameters for poorly studied variable stars from the General Catalog of Variable Stars (G.C.V.S.) (Schmidt 1991b). The selection criteria were (i) stars fainter than the tenth magnitude and (ii) north of the equator stars which were classified as pulsating variables. Schmidt suggested reclassification of 26 stars, 6 of which seem to be eclipsing variables which were previously classified as RR Lyrae stars.

V508 Cyg is one of the new eclipsing variables. The Gen-

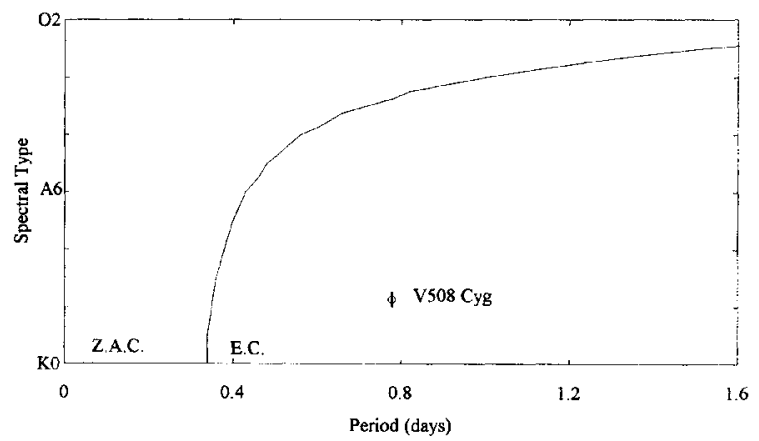

FIG. 1. Period vs spectral-type diagram. The theoretical zero-age contact periods are calculated for $q=1.0$ from the Z. A. M. S. mass-radius relation (Stotters 1972). The critical contact boundary divides the contact systems into zero-age contact systems (Z. A. C.) on the left and evolved contact systems (E. C.) on the right in the figure. The error in period is essentially zero, while the estimated error of \pm 1 subspectral type is shown as a vertical bar. eral Catalog mentions only a couple of references on this system, (Hoffmeister 1949; M.V.S. 1957) which relate to its discovery and early finding charts. There seems to be no other existing literature on V508 Cyg. The shape of the lightcurve and its short orbital period indicates that it is likely to be a contact system. Further evidence in support of this comes from the criteria for potential contact systems (Leung 1990). According to its location in a Period-Spectral-type diagram (Leung \& Schneider 1978), and from our Fig. 1, V508 Cyg could be an evolved contact system. Therefore a detailed photometric study of this system may be of interest to the theory of binary evolution. Thus, we decided to obtain a full phase coverage light curve of this system and derive photometric solutions.

\section{CCD PHOTOMETRY AND OBSERVATIONS}

The observations on V508 Cyg were obtained with a liquid nitrogen cooled CCD photometer on the $0.76 \mathrm{~m}$ telescope at Behlen Observatory, University of Nebraska, on nine nights between 1992 July 28 and November 13. A total of 699 pairs of $V$ - and $R$-band measurements were made to cover most of the light curve.

TABLE 1. Identification data for V508 Cyg.

\begin{tabular}{lcccccc}
\hline \multicolumn{1}{c}{ Star } & G.S.C No. & $\mathrm{V}$ & $\mathrm{R}$ & $\mathrm{V}-\mathrm{R}$ & $\alpha(2000)$ & $\delta(2000)$ \\
\hline V508 Cyg & $3573: 1794$ & 11.99 & $\mathbf{1 1 . 4 3}$ & 0.566 & 203405 & +461616 \\
C1 & $3573: 1541$ & $\mathbf{1 2 . 3 9}$ & $\mathbf{1 1 . 5 6}$ & 0.794 & 203357 & +465117 \\
C2 & $3577: 0181$ & $\mathbf{1 1 . 8 1}$ & $\mathbf{1 1 . 5 2}$ & $\mathbf{0 . 3 0 0}$ & 203415 & +465251 \\
\hline \hline
\end{tabular}


TABLE 2. Observational data for V508 Cyg.

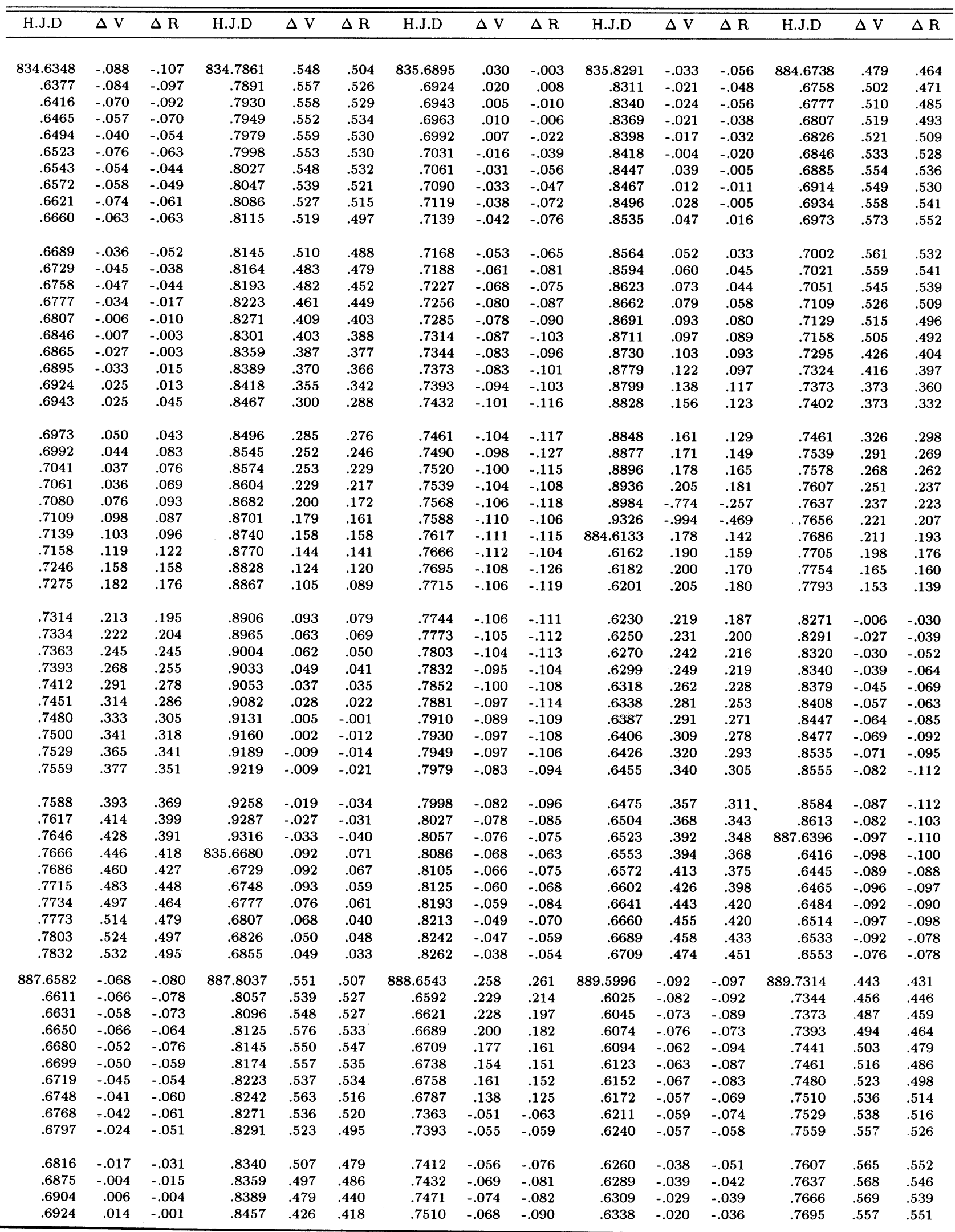


TABLE 2. (continued)

\begin{tabular}{|c|c|c|c|c|c|c|c|c|c|c|c|c|c|c|c|}
\hline H.J.D & $\Delta V$ & \multicolumn{2}{|c|}{$\Delta \mathrm{R}$} & H.J.D & $\Delta V$ & $\Delta \mathrm{R}$ & H.J.D & $\Delta V$ & $\Delta \mathrm{R}$ & H.J.D & $\Delta V$ & $\Delta \mathrm{R}$ & H.J.D & $\Delta \mathrm{V}$ & $\Delta \mathrm{R}$ \\
\hline 6963 & .031 & \multicolumn{2}{|c|}{.005} & .8477 & .400 & .399 & .7529 & -.086 & -.099 & .6367 & -.018 & -.023 & .7734 & .542 & .531 \\
\hline .6992 & .046 & \multicolumn{2}{|c|}{.025} & .8506 & .393 & .398 & .7568 & -.072 & -.083 & .6387 & -.003 & -.015 & .7754 & .543 & .523 \\
\hline .7021 & .053 & \multicolumn{2}{|c|}{.027} & .8535 & .376 & .378 & .7598 & -.079 & -.090 & .6416 & .002 & -.009 & .7783 & .535 & .503 \\
\hline .7041 & .059 & \multirow{2}{*}{\multicolumn{2}{|c|}{$\begin{array}{l}.025 \\
.049\end{array}$}} & .8574 & .368 & .362 & .7617 & -.101 & -.106 & .6436 & .016 & -.003 & .7803 & .527 & .508 \\
\hline .7070 & .065 & & & .8613 & .349 & . 343 & .7646 & -.081 & -.106 & .6465 & .024 & -.005 & .7832 & .512 & .484 \\
\hline .7090 & .075 & & 47 & .8643 & .326 & . 307 & .7686 & -.082 & -.092 & .6484 & $\times 026$ & .011 & .7871 & .472 & .474 \\
\hline .7119 & .082 & & 42 & .8682 & .282 & .285 & .7705 & -.080 & -.080 & .6514 & .029 & .021 & .7900 & .470 & .469 \\
\hline .7158 & .078 & & 66 & .8701 & .283 & .270 & .7734 & -.087 & -.080 & .6533 & .036 & .032 & .7930 & .460 & .434 \\
\hline .7188 & .090 & .0 & 93 & .8730 & .288 & 262 & .7754 & -.103 & -.106 & .6563 & .045 & .042 & .7949 & .422 & .417 \\
\hline .7246 & .117 & .1 & 22 & 888.5742 & .548 & 494 & .7783 & -.103 & -.098 & .6582 & .058 & .043 & .7969 & .413 & .400 \\
\hline .7266 & .139 & & 30 & .5771 & .557 & 493 & .7803 & .102 & -.104 & .6611 & .069 & .067 & .7998 & .401 & .389 \\
\hline .7295 & .160 & .1 & 42 & .5791 & .557 & 530 & .7871 & .092 & -.099 & .6631 & .077 & .061 & .8037 & .374 & .366 \\
\hline .7324 & .186 & & 44 & .5820 & .570 & 528 & .7910 & .098 & -.102 & .6660 & .082 & .083 & .8066 & .358 & .356 \\
\hline .7373 & .174 & .1 & 53 & .5869 & .575 & 500 & .7930 & .096 & -.104 & .6689 & .096 & .090 & .8096 & .340 & .337 \\
\hline .7393 & .188 & .1 & & .5898 & .580 & 525 & .7959 & .106 & -.097 & .6709 & .101 & .095 & .8115 & .322 & .323 \\
\hline .7422 & .204 & .1 & & .5918 & .554 & 536 & .7998 & .100 & -.100 & .6738 & .114 & .100 & .8145 & .321 & .303 \\
\hline .7441 & .220 & .1 & & .5947 & .578 & 538 & .8018 & .102 & -.101 & .6758 & .122 & .120 & .8164 & .285 & .292 \\
\hline .7480 & .240 & .2 & & .5967 & .575 & 538 & .8057 & 102 & -.106 & .6787 & .132 & .127 & .8203 & .273 & .265 \\
\hline .7510 & .260 & .2 & & .5986 & .579 & 528 & .8096 & .100 & -.119 & .6807 & .155 & .144 & .8232 & .256 & .252 \\
\hline .7539 & .276 & .2 & & .6016 & .557 & 543 & .8125 & .090 & -.107 & .6836 & .165 & .151 & .8262 & .244 & .230 \\
\hline .7568 & .283 & .2 & & .6055 & . .546 & 508 & .8154 & .104 & -.104 & .6855 & .159 & .155 & .8291 & .232 & .226 \\
\hline .7588 & .314 & .2 & & .6084 & .541 & 198 & .8174 & .095 & -.116 & .6885 & .181 & .176 & .8330 & .227 & .198 \\
\hline .7617 & .297 & $.2 \varepsilon$ & & .6104 & .526 & 499 & .8203 & .106 & -.112 & .6904 & .179 & .179 & .8350 & .208 & .209 \\
\hline .7666 & .354 & 3 & & .6133 & . 498 & 489 & .8242 & .078 & -.089 & .6934 & .202 & .186 & .8369 & .213 & .179 \\
\hline .7695 & .366 & .35 & & .6162 & .484 & 467 & .8271 & .081 & -.089 & .6953 & .219 & .204 & 398.5967 & -.013 & -.030 \\
\hline .7715 & .371 & .35 & & .6191 & .481 & 462 & .8301 & .068 & -.092 & .7021 & .263 & .229 & .5996 & -.006 & -.021 \\
\hline .7773 & .408 & .38 & & .6211 & .476 & 146 & .8330 & 070 & -.078 & .7051 & .277 & .249 & .6016 & .020 & -.008 \\
\hline .7813 & .426 & $.4]$ & & .6240 & .449 & 130 & 89.5752 & 101 & -.133 & .7070 & .295 & .269 & .6055 & .021 & .003 \\
\hline .7832 & .454 & .42 & & .6270 & .442 & 408 & .5781 & 113 & -.118 & .7100 & .305 & .272 & .6074 & .024 & .010 \\
\hline .7852 & .455 & .43 & 39 & .6299 & .429 & 401 & .5801 & 110 & -.125 & .7119 & .312 & .299 & .6094 & .040 & .022 \\
\hline .7881 & .480 & .46 & & .6318 & .402 & 392 & .5830 & 099 & -.130 & .7148 & .339 & .307 & .6123 & .054 & .025 \\
\hline .7900 & .490 & .46 & & .6348 & .383 & 381 & .5859 & 095 & -.121 & .7168 & .349 & .321 & .6143 & .056 & .026 \\
\hline .7939 & .493 & .46 & & .6367 & .378 & 371 & .5898 & 092 & -.126 & .7197 & .371 & .332 & .6172 & .057 & .041 \\
\hline .7959 & .518 & .48 & & .6426 & .335 & 322 & .5928 & 084 & -.110 & .7236 & .401 & .377 & .6191 & .064 & .047 \\
\hline .7988 & .517 & $.4 \varepsilon$ & & .6455 & .308 & 285 & .5947 & .095 & -.111 & .7266 & .420 & .395 & .6211 & .071 & .072 \\
\hline .8008 & .533 & .51 & & .6514 & .268 & 252 & .5977 & 096 & -.111 & .7295 & .446 & .406 & .6250 & .079 & .057 \\
\hline 898. & 279 & .085 & .069 & 898.7305 & .582 & .559 & 916.6143 & .331 & .292 & 916.7275 & .235 & .192 & 939.6357 & .481 & .436 \\
\hline & 299 & .088 & .078 & .7324 & .576 & .566 & .6182 & .353 & .332 & .7305 & .219 & .174 & .6387 & .505 & .465 \\
\hline & 318 & .098 & .090 & .7354 & .576 & .559 & .6201 & .374 & .349 & .7324 & .206 & .164 & .6406 & .516 & .474 \\
\hline & 338 & .113 & .102 & .7393 & .563 & .547 & .6230 & .408 & .365 & .7363 & .186 & .146 & .6436 & .526 & .479 \\
\hline & 367 & .117 & .100 & .7441 & .536 & .526 & .6260 & .415 & .398 & .7393 & .189 & .166 & .6455 & .532 & .505 \\
\hline & 387 & .121 & .109 & .7471 & .531 & .532 & .6289 & .425 & .413 & .7412 & .166 & .141 & .6484 & .543 & .506 \\
\hline & 406 & .143 & .116 & .7490 & .502 & .514 & .6309 & .431 & .408 & .7432 & .163 & .123 & .6504 & .548 & .505 \\
\hline & 426 & .149 & .125 & .7520 & .516 & .491 & .6348 & .470 & .427 & .7461 & .148 & .119 & .6533 & .550 & .521 \\
\hline & 455 & .151 & .130 & .7539 & .499 & .472 & .6367 & .476 & .444 & .7480 & .139 & .087 & .6553 & .554 & .514 \\
\hline & 494 & .168 & .142 & .7568 & .468 & .461 & .6396 & .482 & .457 & .7529 & .115 & .076 & .6582 & .540 & .513 \\
\hline & 523 & 191 & .163 & .7588 & .462 & .445 & .6416 & .499 & .486 & .7559 & .107 & .061 & .66 & .552 & .538 \\
\hline & 543 & . 190 & .177 & .7637 & .424 & .411 & .6445 & .510 & .473 & .7578 & .097 & .050 & .6641 & .567 & .528 \\
\hline & 572 & .209 & .206 & .7656 & .401 & .410 & .6484 & .552 & .513 & .7607 & .087 & .061 & .6660 & .573 & .519 \\
\hline & 592 & .221 & .209 & .7695 & .390 & .365 & .6514 & .551 & .524 & 939.5625 & .090 & .039 & .6680 & .569 & .528 \\
\hline & $\$ 21$ & .245 & .211 & .7725 & .368 & .344 & .6533 & .544 & .513 & .5645 & .090 & .045 & .6709 & .555 & .514 \\
\hline & $\$ 41$ & .262 & .222 & .7754 & .353 & .326 & .6572 & .550 & .538 & .5664 & .096 & .054 & .6729 & .523 & .525 \\
\hline & 660 & .259 & .233 & .7783 & .325 & .311 & .6621 & .554 & .510 & .5703 & .117 & .072 & .6758 & .526 & .490 \\
\hline & 889 & .270 & .252 & .7813 & .328 & .295 & .6670 & .556 & .514 & .5732 & .139 & .087 & .6787 & .513 & .483 \\
\hline & 09 & .279 & .267 & .7832 & .297 & .288 & .6709 & .543 & .513 & .5762 & .146 & .099 & .6816 & .502 & .472 \\
\hline & 29 & .301 & .270 & .7871 & .275 & .248 & .6738 & .538 & .504 & .5781 & .150 & .125 & .6836 & .476 & .461 \\
\hline & 58 & 306 & .293 & .7891 & .258 & .245 & .6758 & .527 & .504 & .5820 & .180 & .141 & .68 & .466 & .447 \\
\hline & 97 & .326 & .299 & .7920 & .252 & .220 & .6777 & .507 & .477 & .5840 & .177 & .149 & .6885 & .439 & .426 \\
\hline & 36 & .351 & .324 & .7939 & .232 & .207 & .6807 & .513 & .498 & .5869 & .201 & .155 & .6904 & .462 & .417 \\
\hline & 355 & .365 & .337 & .7959 & .205 & .205 & .6836 & .493 & .486 & .5889 & .208 & .167 & .6943 & .405 & .408 \\
\hline & 85 & .382 & .360 & 916.5762 & .127 & .085 & .6855 & .487 & .472 & .5908 & .212 & .179 & .6973 & .411 & .377 \\
\hline & 04 & .387 & .356 & .5781 & .146 & .096 & .6875 & .467 & .439 & .5947 & .249 & .202 & .7002 & .375 & .359 \\
\hline & 24 & .402 & .367 & .5811 & .168 & .104 & .6904 & .462 & .443 & .5977 & .267 & .242 & .7031 & .366 & .326 \\
\hline & 63 & .417 & .407 & .5830 & .173 & .124 & .6924 & .438 & .412 & .6016 & .299 & .238 & .7061 & .363 & .308 \\
\hline
\end{tabular}


TABLE 2. (continued)

\begin{tabular}{|c|c|c|c|c|c|c|c|c|c|c|c|c|c|c|}
\hline H.J.D & $\Delta V$ & $\Delta \mathrm{R}$ & H.J.D & $\Delta \mathrm{V}$ & $\Delta \mathrm{R}$ & H.J.D & $\Delta \mathrm{V}$ & $\Delta \mathrm{R}$ & H.J.D & $\Delta \mathrm{V}$ & $\Delta R$ & H.J.D & $\Delta V$ & $\Delta \mathrm{R}$ \\
\hline .6992 & .446 & .415 & .5850 & .182 & .125 & .6953 & .421 & .396 & .6045 & .302 & .267 & .7080 & .320 & .294 \\
\hline .7012 & .453 & .439 & .5869 & .204 & .126 & .6973 & .404 & .383 & .6064 & .321 & .269 & .7109 & .326 & .295 \\
\hline .7031 & .476 & .434 & .5908 & .208 & .175 & .7002 & .399 & .366 & .6094 & .314 & .277 & .7139 & .304 & .272 \\
\hline .7061 & .474 & .446 & .5938 & .216 & .176 & .7031 & .380 & .366 & .6123 & .329 & .285 & .7158 & .311 & .234 \\
\hline .7080 & .486 & .471 & .5967 & .229 & .197 & .7051 & .361 & .350 & .6152 & .344 & .316 & .7188 & .276 & .253 \\
\hline .7109 & .521 & .477 & .5986 & .248 & .191 & .7080 & .356 & .323 & .6172 & .365 & .328 & .7207 & .257 & .225 \\
\hline .7139 & .547 & .537 & .6006 & .257 & .226 & .7100 & .330 & .312 & .6201 & .385 & .344 & .7227 & .238 & .200 \\
\hline .7168 & .535 & .524 & .6025 & .280 & .244 & .7119 & .323 & .306 & .6221 & .391 & .357 & .7256 & .250 & .219 \\
\hline .7188 & .571 & .519 & .6055 & .274 & .247 & .7148 & .288 & .282 & .6270 & .420 & .371 & .7275 & .236 & .177 \\
\hline .7207 & .549 & .547 & .6074 & .290 & .261 & .7178 & .297 & .253 & .6289 & .434 & .388 & .7305 & .211 & .189 \\
\hline .7236 & .563 & .552 & .6094 & .316 & .276 & .7207 & .282 & .268 & .6309 & .447 & .408 & .7324 & .198 & .191 \\
\hline .7266 & .569 & .544 & .6123 & .325 & .301 & .7236 & .261 & .246 & .6338 & .463 & .413 & & & \\
\hline
\end{tabular}

${ }^{\text {a Heliocentric Junlian date is } 2,448,000+\text { H.J.D }}$

The CCD photometric system and observational techniques have been described previously (Schmidt 1988, 1991a; Schmidt et al. 1990). The extraction of differential magnitudes using the existing software on the Micro Vax II necessitated only minor changes in the observing scheme from that employed by Schmidt for his variable star survey.

For differential magnitudes we have used the same comparison stars as Schmidt (1991b). Since these comparison stars are within a few arcmin of the variable, accurate differential magnitudes can be derived even under inferior photometric conditions. Table 1 lists the Guide Star catalog identification numbers and coordinates for V508 Cyg and the comparison stars. The mean magnitude and color of the variable and the adopted magnitudes and colors of the comparison stars are also given (Schmidt 1993). A finder chart is published elsewhere (Schmidt \& Reiswig 1993).

The extinction coefficients were obtained using one of the comparison stars as the extinction star. Average color terms from the past few years were adopted. The probable errors in $V$ and $R$ for the comparison stars are 15 and 29 millimagnitudes, respectively. Table 2 lists the heliocentric Julian Dates and differential $V$ and $R$ magnitudes for V508 Cyg (with respect to the mean of the comparison stars). Our observations show that V508 Cyg is a W UMa-type system with a continuously changing brightness and no indication of total eclipse in either the primary or the secondary minimum.

\subsection{Period}

Schmidt's (1993) light curve for V508 Cyg is based on 19 observations each in $V$ and $R$. From it, he derived the following light elements:

Minimum(I)=H.J.D. $2447774.85+0{ }^{\mathrm{d}} 77969( \pm 2) E$.

Using the method of Kwee \& Van Woerden (1956), we have determined eight times of minimum from our data in each filter band. The times of minimum, their probable errors, and weights are collected in Table 3 . We utilized Schmidt's epoch [Eq. (1)] in our calculation of the new light elements. The method of generalized least squares was applied to the times of these minimum (Table 3), to obtain the following light elements for V508 Cyg:

$$
\begin{aligned}
\operatorname{Minimum}(\mathrm{I})= & \text { H.J.D. } 2448888.5923( \pm 3) \\
& +0^{\mathrm{d}} 7796587( \pm 7) E .
\end{aligned}
$$

Equation (2) shows that the new period differs from that in Eq. (1) by $0.00003(\simeq 2.6 \mathrm{~s})$ days. The differences between the observed and the computed times of minima $(\mathrm{O}-\mathrm{C})$ are listed in Table 3. Figure 2 shows the plot of $(\mathrm{O}-\mathrm{C})$ vs $E$ (cycles) for V508 Cyg. The results do not give any evidence of a change in period, keeping in mind that the one point

\begin{tabular}{|c|c|c|c|c|c|c|c|c|c|}
\hline \multicolumn{5}{|c|}{$\mathrm{V}(5500 \AA)$} & \multicolumn{5}{|c|}{$\mathrm{R}(7000 \AA)$} \\
\hline H.J.D. & P.E. & Wt. & $\mathrm{E}$ & $\mathrm{O}-\mathrm{C}$ & H.J.D. & P.E. & Wt. & $\mathrm{E}$ & $\mathrm{O}-\mathrm{C}$ \\
\hline $7774.8500^{*}$ & $\ldots$ & 06 & -1428.5 & 0.0002 & & & & & \\
\hline 8834.7945 & 0.00053 & 07 & -0069.0 & -0.0013 & 8834.7946 & 0.00033 & 08 & -0069.0 & -0.0012 \\
\hline 8884.6923 & 0.00040 & 08 & -0005.0 & -0.0017 & 8884.6924 & 0.00037 & 08 & -0005.0 & -0.0016 \\
\hline 8887.8134 & 0.00055 & 07 & -0001.0 & 0.0008 & 8887.8140 & 0.00097 & 04 & -0001.0 & 0.0014 \\
\hline 8888.5912 & 0.00068 & 06 & 0000.0 & -0.0011 & 8888.5999 & 0.00158 & 01 & 0000.0 & 0.0076 \\
\hline 8889.7624 & 0.00011 & 10 & 0001.5 & 0.0007 & 8889.7633 & 0.00127 & 02 & 0001.5 & 0.0016 \\
\hline 8898.7279 & 0.00065 & 06 & 0013.0 & 0.0001 & 8898.7281 & 0.00055 & 07 & 0013.0 & 0.0003 \\
\hline 8916.6618 & 0.00036 & 08 & 0036.0 & 0.0018 & 8916.6622 & 0.00048 & 07 & 0036.0 & 0.0022 \\
\hline 8939.6589 & 0.00074 & 06 & 0065.5 & -0.0010 & 8939.6591 & 0.00049 & 07 & 0065.5 & -0.0008 \\
\hline
\end{tabular}

TABLE 3. Times of minima for V508 Cyg.

*Adopted from the epoch of the Schmidts (1993) light elements. 


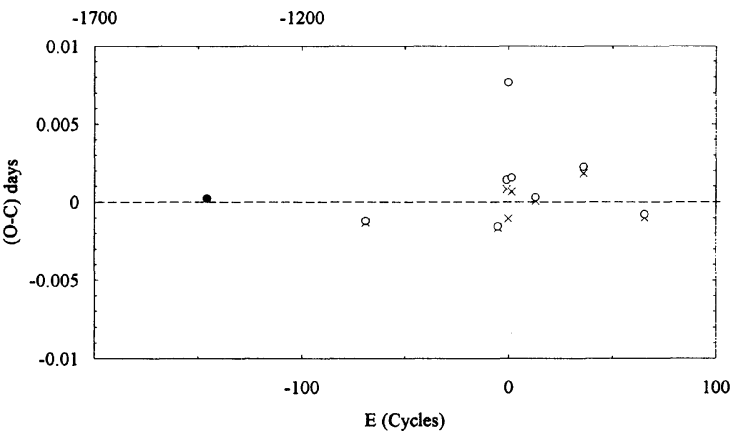

FIG. 2. (O-C) vs elapsed cycles. The filled circle (cycles on the top axis) represents Schmidt's observation. The open circles represent our $V$ observations, while the crosses represent the $R$ observations (cycles are on the bottom axis).

which has the high $(\mathrm{O}-\mathrm{C})$ is the one with a large probable error (see Table 3).

\subsection{Photometric Analysis}

The latest 1993 version of the Wilson-Devinney program (W-D) (first described by Wilson \& Devinney 1971) was used for our photometric analysis. About 80 normal points were formed for each light curve with weights proportional to the number of points used to form each normal point. After making rough estimates of the photometric parameters with the lightcurve program, the analysis was started with the W-D differential correction program. Both $V$ and $R$ light curves were employed simultaneously in deriving the solutions. In the W-D notation, star 1 is eclipsed at the primary minimum. The analysis started with a detached binary configuration (mode 2 in the W-D code). The temperature $\left(T_{1}\right)$ of the hotter star was obtained from the color of the system assuming no reddening (see Table 1), and the program was

TABle 4. Photometric solutions for V508 Cyg.

\begin{tabular}{lrr}
\hline \multicolumn{1}{c}{ Parameter } & First Solution & Second Solution \\
\hline $\mathrm{q}\left(\mathrm{m}_{2} / \mathrm{m}_{1}\right)$ & $0.4431 \pm 0.0043$ & $1.1915 \pm 0.0037$ \\
$\mathrm{~L}_{1} /\left(\mathrm{L}_{1}+\mathrm{L}_{2}\right)(5500 \AA)$ & $0.6436 \pm 0.0039$ & $0.4704 \pm 0.0040$ \\
$\mathrm{~L}_{1} /\left(\mathrm{L}_{1}+\mathrm{L}_{2}\right)(7000 \AA)$ & $0.6470 \pm 0.0037$ & $0.4689 \pm 0.0037$ \\
$i$ & $78^{\circ} .30 \pm 0.14$ & $76^{\circ} .29 \pm 0.09$ \\
$\Omega_{1}=\Omega_{2}$ & $2.5771 \pm 0.0079$ & $3.7445 \pm 0.0041$ \\
$\Omega(\text { in })^{* *}$ & 2.7646 & 4.0528 \\
$\Omega(\text { out })^{* *}$ & 2.4968 & 3.4940 \\
$f(\%$ of overflow $)$ & $70.01 \%$ & $55.17 \%$ \\
$\mathrm{~A}_{1}=\mathrm{A}_{2}{ }^{*}$ & 0.50 & 0.50 \\
$x_{1}=x_{2}{ }^{*}$ & 0.60 & 0.60 \\
$g_{1}=g_{2}^{*}$ & 0.30 & 0.30 \\
$\mathrm{r}_{1}$ (pole) & $0.4599 \pm 0.0008$ & $0.3801 \pm 0.0006$ \\
$\mathrm{r}_{1}$ (side) & $0.4999 \pm 0.0011$ & $0.4066 \pm 0.0008$ \\
$\mathrm{r}_{1}$ (back) & $0.5452 \pm 0.0012$ & $0.4685 \pm 0.0018$ \\
$\mathrm{r}_{2}$ (pole) & $0.3286 \pm 0.0039$ & $0.4088 \pm 0.0008$ \\
$\mathrm{r}_{2}$ (side) & $0.3499 \pm 0.0051$ & $0.4391 \pm 0.0011$ \\
$\mathrm{r}_{2}$ (back) & $0.4257 \pm 0.0148$ & $0.4947 \pm 0.0020$ \\
$\mathrm{~T}_{1} \mathrm{~K}^{\circ}$ & $5600^{*}$ & $5600^{*}$ \\
$\mathrm{~T}_{2} \mathrm{~K}^{\circ}$ & $5688 \pm 7$ & $5565 \pm 6$ \\
& &
\end{tabular}

\footnotetext{
* Assumed ** Theorectical values
}

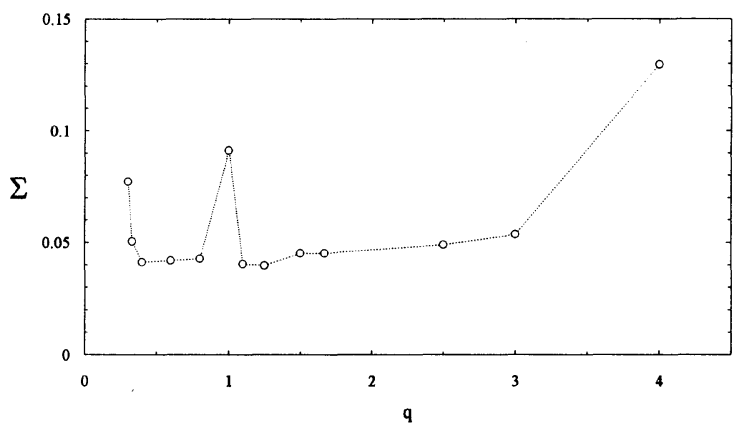

FIG. 3. Sum of the residuals vs mass ratio for V508 Cyg. Each open circle represents a solution.

allowed to adjust the following parameters: inclination $(i)$ of the orbit, temperature $\left(T_{2}\right)$ of the cooler star, nondimensional gravity potentials $\left(\Omega_{1}\right.$ and $\left.\Omega_{2}\right)$ of two stars, mass ratio $\left(q=m_{2} / m_{1}\right)$ of the system, and luminosity $\left(L_{V, 1}\right.$ and $\left.L_{R, 1}\right)$ of the hotter star (that of the cooler star is computed by assuming blackbody atmosphere). The values for the limb darkening coefficients $\left(x_{1}\right.$ and $\left.x_{2}\right)$ were interpolated from the tables of Al-Naimy (1978). Albedos $\left(A_{1}\right.$ and $\left.A_{2}\right)$ and gravity darkening coefficients $\left(g_{1}\right.$ and $\left.g_{2}\right)$ for convective atmospheres were assumed.

After many runs the system converged toward a contact configuration (mode 3 ). This was tested by starting the solution as a semidetached configuration (mode 4 in the W-D code) and again the solution converged toward a contact configuration. Having established a contact configuration for the system, we proceeded with the mode 3 configuration for all further solutions. The next step was to find a global value of the mass ratio from a " $q$ " search procedure. In this search,13 localized solutions were obtained for discrete fixed values of the mass ratio. The results are displayed in Fig. 3.

As one can see from the Fig. 3 there are unfortunately two sets of photometric parameters which give essentially the same good fit to the observations: One with a mass ratio of 0.40 and the other with a value of 1.15. The former solution gives us an A-type contact system (primary eclipse occurs at transit), while the latter solution gives us a W-type contact system (primary eclipse occurs at occultation). The next step in the analysis was to investigate solutions around the mass ratios of 0.40 and 1.15 , which have the smallest sum of the residuals shown in Fig. 3. However, this time we allowed the mass ratio to be a free parameter. Thus two sets of photometric parameters were found for mass ratios of 0.443 and 1.192 (see Table 4). As a final step in the analysis, the values of limb darkening, albedo, and gravity darkening were treated as free parameters to see if adjusting them would improve the two solutions. All trials did not show any convergence or an improvement in the sum of residuals over what we had obtained earlier by keeping the values fixed. Thus, the two sets of solutions with mass ratio of 0.443 and 1.192 were adopted as the photometric solutions for V508 Cyg.

The computed light curves are shown as continuous curves in Figs. 4 and 5 for the final two solutions while Table 


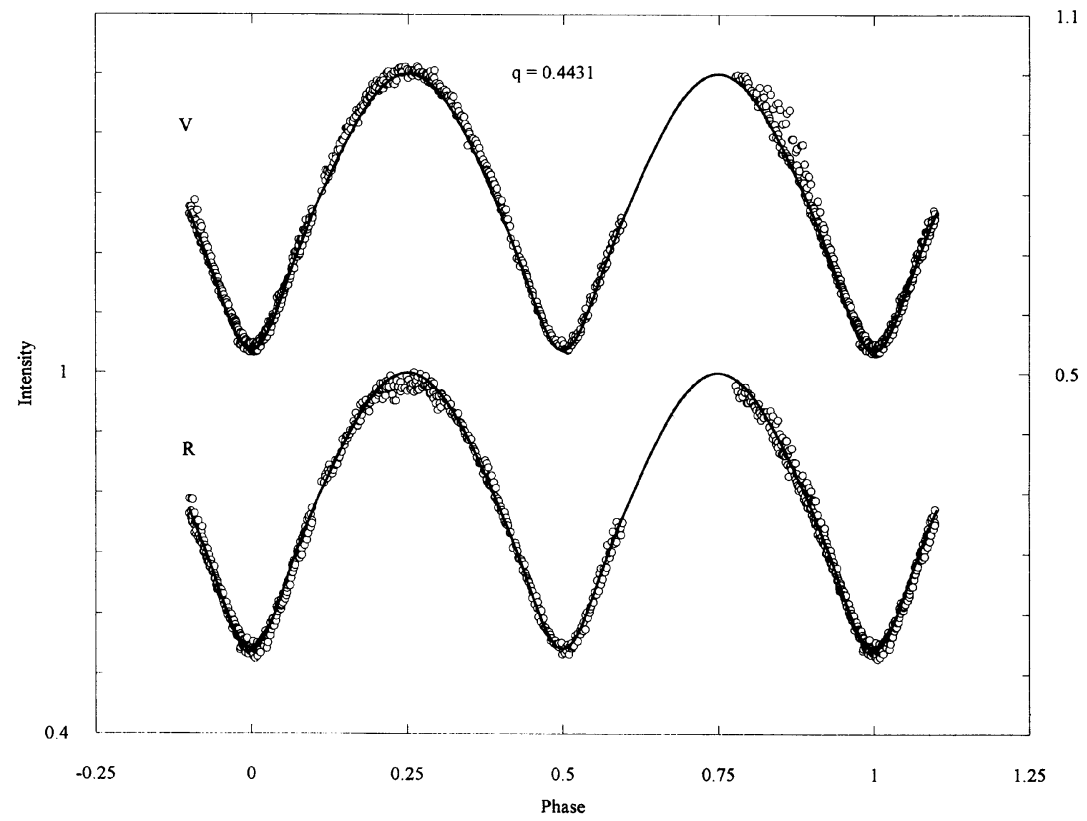

FIG. 4. Observed and computed light curves for V508 Cyg, $q=0.4431$. The circles show individual observations while the continuous curve is the computed curve.

4 describes them. For both solutions the agreement between the theoretical and observed light curve is good. In both of the solutions the temperature difference between the components is less than $100 \mathrm{~K}$. This determination of differences in temperature in eclipsing systems is very reliable since these are directly related to eclipse depths. The primary minimum is only slightly deeper than the secondary by about $0 .{ }^{\mathrm{m}} 009$ for $V$ bandpass and $0{ }^{\mathrm{m}} 014$ for $R$ bandpass. Such small differences demonstrate that the components are of similar color (see Table 1). Assuming no reddening, both compo-

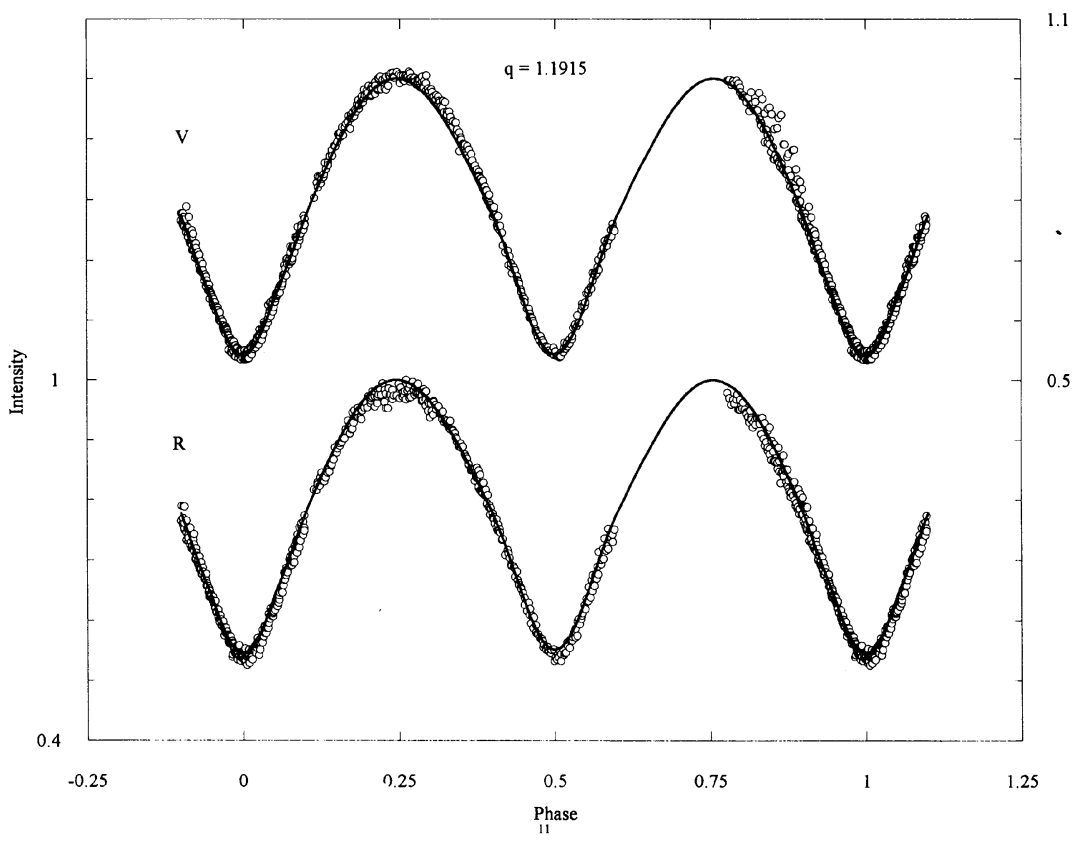

FIG. 5. Observed and computed light curves for V508 Cyg, $q=1.1915$. The circles show individual observations while the continuous curve is the computed curve. 


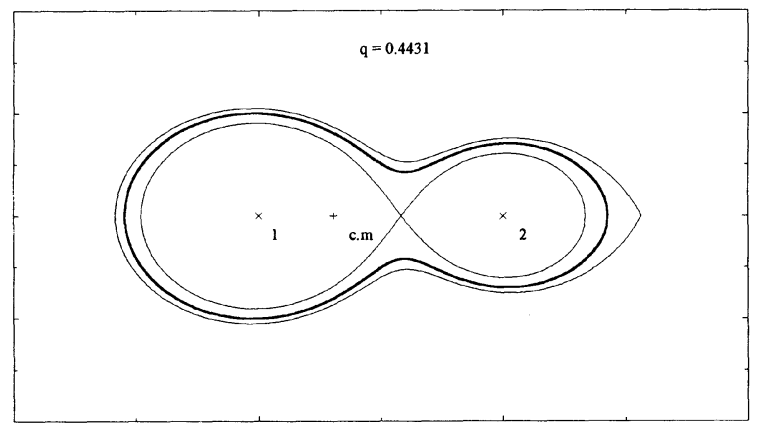

FIG. 6. Binary configuration for V508 Cyg as an A-type W UMa system with $q=0.4431$.

nents have a spectral type of about G5. The orbital inclinations found for both the solutions are small $\left(i \simeq 76^{\circ}\right)$ which makes it more difficult to determine the values of limb darkening, albedo, and gravity darkening. However, as can be seen, the solution at $q=1.19$ has a shallower contact $(f$ $=55 \%$ ) than at $q=0.44$, where the contact is $f=70 \%$. Table 4 also shows that for both the solutions the two stars have similar luminosities, suggesting that V508 Cyg should be a doubled lined spectroscopic binary. Solution 1 in Table 4 suggests that the temperature of the secondary may be slightly higher, however, the difference in eclipse minimum from the computed curve is within the observational scatter of the observed data. The configurations are shown in Figs. 6 and 7. It is believed that spectroscopic study of this system will settle the uniqueness of mass ratio and yield more information on the system.

\section{DISCUSSION}

We believe that evolved contact systems start out from a detached configuration and evolve into a semidetached system and in some cases eventually become contact due to evolution of the massive component (see for example, de Loore \& Doom 1992; Guinan \& Giménez 1993). For relatively close systems, the mass transfer phase takes place during the core $\mathrm{H}$-burning phase; such systems are referred to as case A-type mass transfer (Kippenhahn \& Weigert 1967; Plavec 1968; Paczyński 1971). Most of the evolved contact systems discovered so far are case A-type systems. It is believed that this is the result of observational selection effect

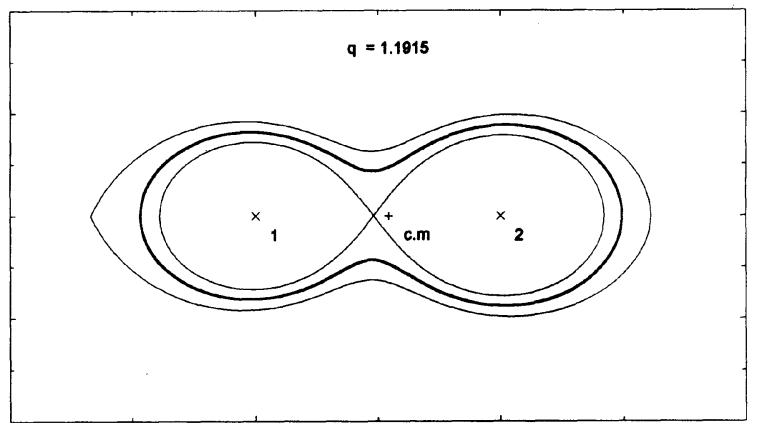

FIG. 7. Binary configuration for V508 Cyg as a B-type W UMa system with $q=1.1915$.

(short period systems are often selected for observation). These systems should lie relatively close to the main sequence in an $\mathrm{H}-\mathrm{R}$ diagram. With respect to the periodspectral-type diagram, they will be found in the evolved contact domain and relatively close to the zero-age critical contact boundary (e.g., Fig. 1). On the other hand, for wider binaries the mass transfer phase takes place during the shell H-burning phase of stellar evolution. Such pairs are called case B mass transfer systems. Since these systems are derived from wider pairs, their periods would be longer. Thus, these systems will be located relatively farther away from the zero-age critical contact boundary in the period-spectral-type diagram as shown in Fig. 1 for the case of V508 Cyg. For this reason we believe that this system may be a evolved contact under case $\mathrm{B}$ mass transfer.

The research described here was supported in part by the National Science Foundation through Grant No. AST8815806. The reduction of data was carried out using the facilities of the Minnich Astronomical Computing Center which was funded by a donation from Commander Charles B. Minnich. S. G. would like to thank his parents for providing the 486 PC computer which was extensively used for analysis work. The authors wish to thank Donald J. Taylor for his help in keeping the observatory in good working order while the observations were carried out. Thanks are also due to R. E. Wilson for making available the 1993 version of the Wilson-Devinney model which can very easily be adopted to run on PC computers. Finally, the authors would like to thank the anonymous referee for careful editorial comments.

\section{REFERENCES}

Al-Naimy, H. M. 1978, Ap\&SS, 53, 181

de Loore, C. W. H., \& Doom, C. 1992, Astrophysics and Space Science Library (ASSL), Structure and Evolution of Single and Binary Stars (Kluwer, Dordrecht), Vol. 179

Guinan, E. F., \& Giménez, A. 1993, Astrophysics and Space Science Library (ASSL), in The Realm of Interacting Binary Stars, edited by J. Shade, Jr., G. McCluskey, and Y. Kondo (Kluwer, Dordrecht), Vol. 177, p. 51

Hoffmeister, C. 1949, Veroff. Stern. Sonneberg (VSS), No. 3, p. 1

Kippenhahn, R., \& Weigert, A., 1967, ZAp, 65, 251

Kwee, K. K., \& Van Woerden H. 1956, Bull. Astron. Inst. Netherlands, 12, 327
Leung, K. C. 1990, NATO ASI Series, C319, in Active Close Binaries, edited by C. Ibanoğlu (Kluwer, Dordrecht), p. 881

Leung, K. C., \& Schneider, D. P. 1978, ApJ, 222, 917

M. V. S. 1957, Mitt. über veränderliche Sterne, Berlin-Babelsberg und Sonneberg, No. 327

Paczyński, B. 1971, ARA\&A, 9, 183

Plavec, M. 1968, Advan. Astron. Astrophys., 6, 201

Schmidt, E. G. 1988, in Automated Small Telescopes, edited by D. S. Hayes and R. M. Genet (Fairborn Press, Mesa), p. 195

Schmidt, E. G. 1991a, in Proceedings of the ASP Symposium, Robotic Observatories: Present and Future, July, edited by S. Baliunas and J. L. 
Richard Boston (Fairborn Press, Mesa), p. 213 Schmidt, E. G. 1991b, AJ, 102, 1766

Schmidt, E. G. 1993, private communications

Schmidt, E. G., \& Reiswig, D. E. 1993 AJ, 106, 2429
Schmidt, E. G., Loomis, C. G. Groebner, A. T., \& Potter, C. T. 1990, ApJ, 360,604

Stotters, R. 1972, ApJ, 175, 431

Wilson, R. E., \& Devinney, E. J. 1971, ApJ, 166, 605 